\title{
Study on the temperature field loaded by a shaped laser beam on the top surface of a cylinder head for thermal fatigue test
}

\section{S.-Z. Nie}

nieshuzhen@aoe.ac.cn

\section{J. Yu}

G. Yu

Q.-F. Tan

Z.-W. Fan

\begin{abstract}
Department of Optoelectronic Engineering, Academy of Opto-Electronics, Chinese Academy of Sciences, No. 9 Dengzhuang south Road, Haidian District, Beijing 100094, People's Republic of China Department of Optoelectronic Engineering, Academy of Opto-Electronics, Chinese Academy of Sciences, No. 9 Dengzhuang south Road, Haidian District, Beijing 100094, People's Republic of China Key Laboratory of Mechanics in Advanced Manufacturing, Institute of Mechanics, Chinese Academy of Sciences, No. 15 Beisihuan west Road, Haidian District, Beijing 100190, People's Republic of China State Key Laboratory of Precision Measurement Technology and Instruments, Tsinghua University, Beijing 100084, People's Republic of China

Department of Optoelectronic Engineering, Academy of Opto-Electronics, Chinese Academy of Sciences, No. 9 Dengzhuang south Road, Haidian District, Beijing 100094, People's Republic of China
\end{abstract}

In thermal fatigue test, the key point is whether the temperature field on the top surface of cylinder head induced by the heat source can well match it in real service. In order to produce the target temperature field in service which is measured by thermocouples, shaped laser beam generated by diffractive optics element (DOE) is chosen as the heat source to irradiate on the top surface of cylinder head. The DOE is designed based on the Gerchberg-Saxton (CS) algorithm and the simulated temperature field is calculated by finite element model (FEM). The results show that the simulated and experimental temperature field can well match the target one which demonstrates that this method is feasible to produce the target temperature field and can be used in thermal fatigue test.

[DOI: http://dx.doi.org/10.2971/jeos.2014.14038]

Keywords: Temperature field, shaped laser beam, diffractive optics element, thermal fatigue test

\section{INTRODUCTION}

During diesel engine works in service, as one of the key parts, cylinder head is subjected to thermal fatigue due to periodic thermal loading of high temperature combustion gas, which will lead to intense stress concentration and accelerate the failure or fault of cylinder head [1]. With the development of high power density diesel engine, the failure and fault of cylinder head caused by thermal fatigue have become a main problem [2]. Therefore, several approaches with different heat sources to perform thermal fatigue test on cylinder head have been proposed, such as localized flame, high frequency wire coil, quartz lamp, etc. [3, 4]. The accuracy and reliability of thermal fatigue test depends on whether the temperature field on the top surface of cylinder head induced by the heat source can well be consistent with it in real service condition. Because the temperature field in real service condition is non-uniform and always has particular distribution [5], it is difficult with these heat sources to generate the required temperature field.

Laser has temporal and spatial controllable characteristic, so it can be considered as the heat source to irradiate on the top surface of cylinder head to perform thermal fatigue test [6]. Recently, the shaped high power laser beam with symmetric distribution has been employed in thermal fatigue test on large engine parts like pistons and cylinder heads [7]-[9]. However, the most studies focus on pistons and the temperature field on cylinder head induced by the shaped laser beam with sym- metric distribution can't well reflect the real temperature field in service which always is non-uniform and non-symmetric.

The aim of the present paper is that the temperature field on the top surface of cylinder head loaded by shaped laser beam with certain intensity distribution can well match the target one in real service. The temperature field on the top surface of cylinder head in work conditions is measured by thermocouples and it is assumed to be the target temperature distribution. The shaped laser beam is produced with the aid of diffractive optics element (DOE) which can modulate freely the irradiance and phase profile of laser beam using their surface relief microstructures [10]. Before a DOE can be manufactured, the temperature field loaded by the shaped laser beam can be calculated based on finite element model (FEM) [11]-[13]. When the simulated temperature distribution well agrees with the target one, the DOE can be designed according to the certain intensity distribution of the shaped laser beam and then can be fabricated by very large scale integration technique [14]. Finally, the temperature test experiment on the top surface of cylinder head loaded by the shaped laser beam produced by the DOE is done. The results show that the test temperature field is well consistent with the target one in service. Based on the conclusion, typical thermal loading conditions $[15,16]$, such as thermal high cycle fatigue and thermal low cycle fatigue, can be simulated by controlling the laser 


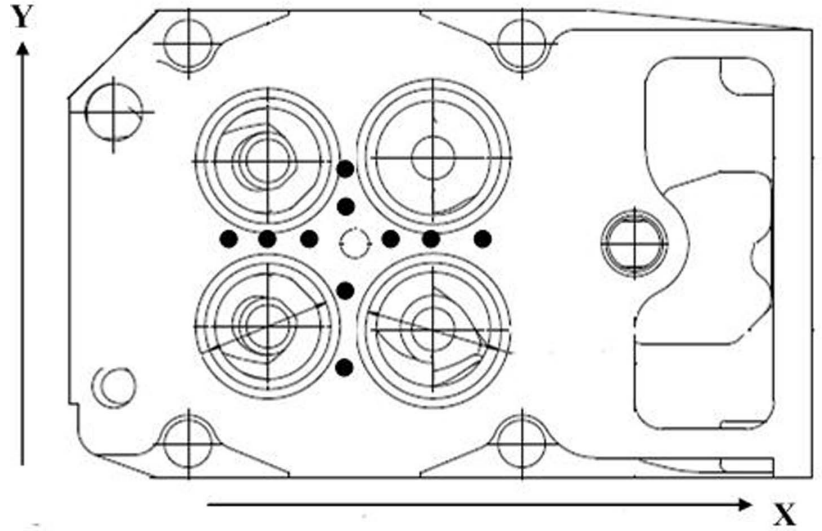

FIG. 1 The thermocouple distributions on the top surface of cylinder head.

loading time or the temperature fluctuation range. The study will provide meaningful technical reference for the evaluation of laser thermal loading and damage on cylinder head.

\section{THE TARGET TEMPERATURE FIELD AND NUMERICAL MODEL}

\subsection{The target temperature field}

The engine parts are under complex thermal mechanical loading in real working conditions, and the real temperature field on the top surface of cylinder head in service is measured by thermocouples. The thermocouples are buried on the bridge zones of the fire board and location of the thermocouples is shown is Figure 1. The distribution of the temperature test spots is among the bridge zones, because the bridge zones are the key regions with particular concerns and always have the risk of damage [17]. Therefore, the temperature test results can well reflect the temperature field on the concerned regions.

The temperature test results by thermocouples which tend towards stability are shown in Figure 2. Figure 2 shows that the three working conditions of $1700 \mathrm{r} / \mathrm{min}(30 \mathrm{KW}), 1900 \mathrm{r} / \mathrm{min}$ (33 KW) and $2000 \mathrm{r} / \mathrm{min}$ (37 KW) have the similar temperature curve, and they are adopted as the target test conditions in thermal fatigue test in our study. The temperature field distribution of the combustion surface is drawn in Figure 3 according to the temperature test results. Figure 3 describes the real temperature field in service which is assumed to be the target temperature distribution. The temperature field on the top surface of cylinder head induced by the shaped laser beam must agree with it, and this is the first step to perform the thermal fatigue test.

\subsection{Numerical model}

In order to calculate the temperature field loaded by the shaped laser beam based on finite element model (FEM), the numerical model of the cylinder head must be built which is shown in Figure 4. The calculated software is ANSYS with the version of 12.0. The mesh type is solid 70 and the total number of nodes is 200983. The power of the input laser beam is $3 \mathrm{~kW}$ and the wavelength is $1064 \mathrm{~nm}$. The transient temperature field $T(x, y, z, t)$ in cylinder head obeys the equation [18].
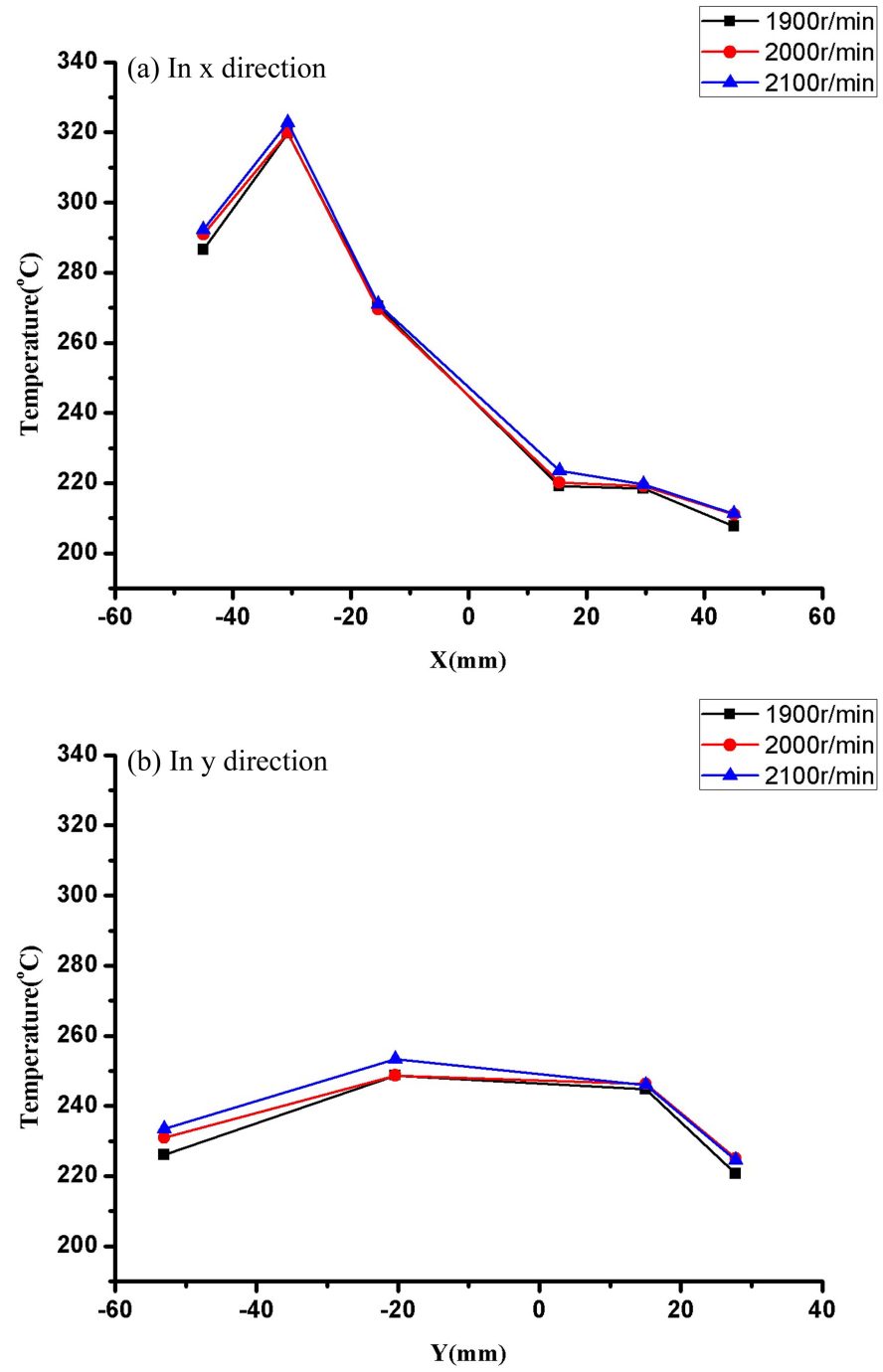

FIG. 2 The temperature test results using thermocouples. (a) The temperature curve in $x$ direction; (b) The temperature curve in $y$ direction.

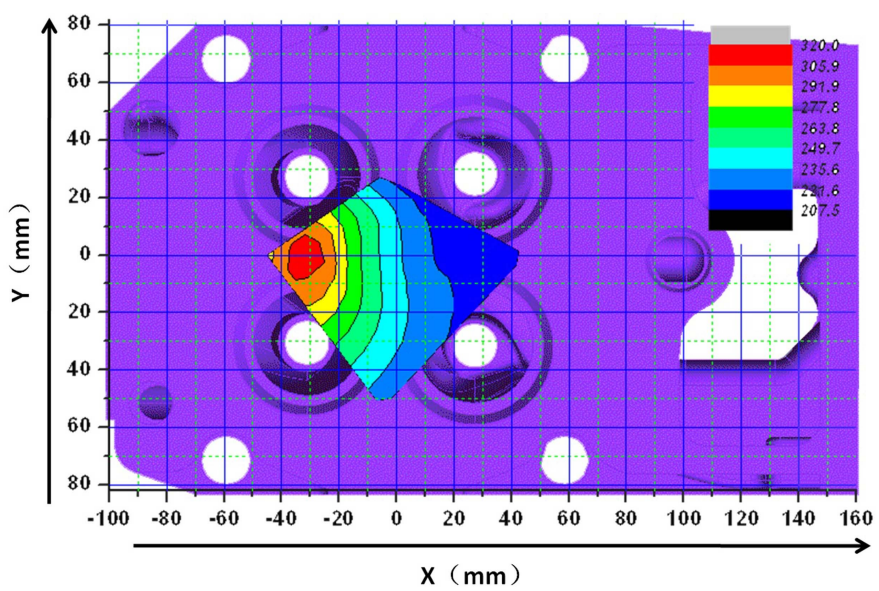

FIG. 3 The temperature field distribution on the combustion surface.

$$
\rho c \frac{\partial T}{\partial t}=k\left(\frac{\partial^{2} T}{\partial x^{2}}+\frac{\partial^{2} T}{\partial y^{2}}+\frac{\partial^{2} T}{\partial z^{2}}\right)
$$

where $\rho, c$, and $k$ are the density, specific heat and thermal conductivity, respectively. Based on Fourier's law of heat conduction, the heat flux on the top surface of cylinder head obeys.

$$
q=-k \frac{\partial T}{\partial n}
$$




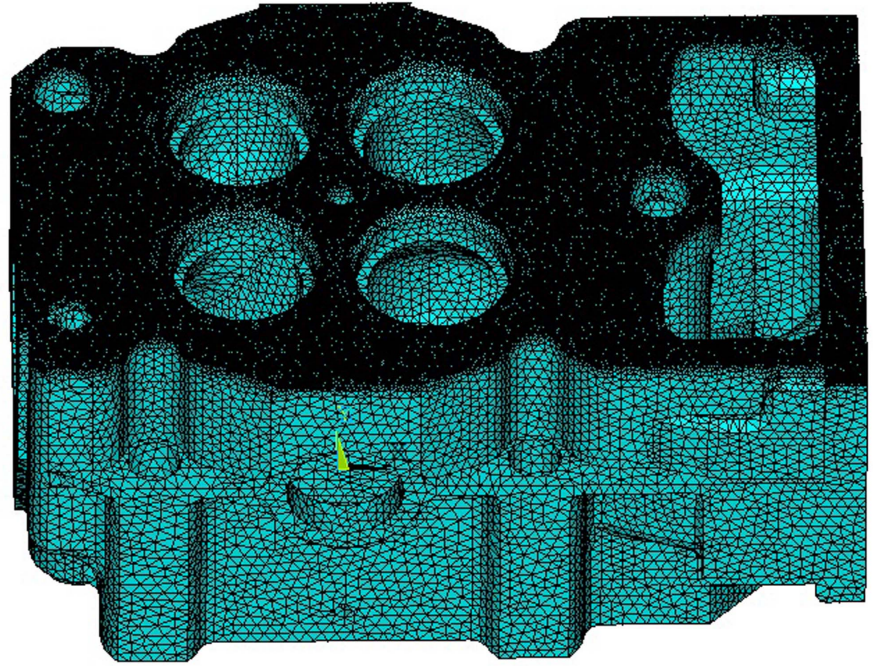

FIG. 4 Finite element model of cylinder heat.

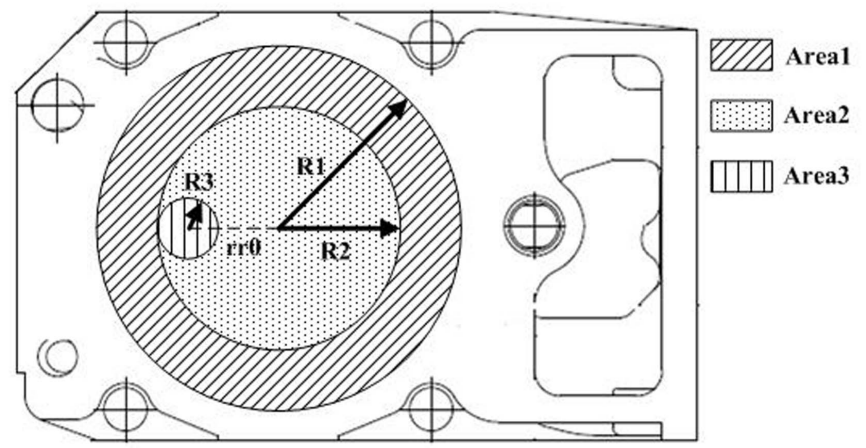

FIG. 5 The final intensity distribution of the shaped laser beam.

where $q$ is the heat flux density vector, $n$ represents normal directions to the surface, and $\partial T / \partial n$ means the temperature gradient. The plane of bottom surface is assumed to be thermal insulation, so it can be assumed adiabatic boundary conduction.

$$
-\left.k \frac{\partial T}{\partial n}\right|_{\Gamma}=0
$$

Surfaces besides the bottom surface are assumed to be natural convection with the environment.

$$
-\left.k \frac{\partial T}{\partial n}\right|_{n}=h_{1}\left(T-T_{a}\right)
$$

where $h_{1}$ is the coefficient of convection, $T_{a}$ is the environmental temperature.

The material of the cylinder head in our study is HT250. In order to make the experimental and simulated results agree with each other, the model parameters have been verified [19]. The temperature fluctuation range on the top surface of cylinder head in service is below $350^{\circ} \mathrm{C}$, so the parameters of the model can be simplified to be invariant. The parameters after verification are shown as below: density is $7.3 \mathrm{~g} / \mathrm{cm}^{3}$, specific heat is $0.54 \mathrm{~J} /\left(\mathrm{g} \cdot{ }^{\circ} \mathrm{C}\right)$, thermal conductivity is $52 \mathrm{~W} /\left(\mathrm{m} \cdot{ }^{\circ} \mathrm{C}\right)$, and absorption rate is $50 \%$.
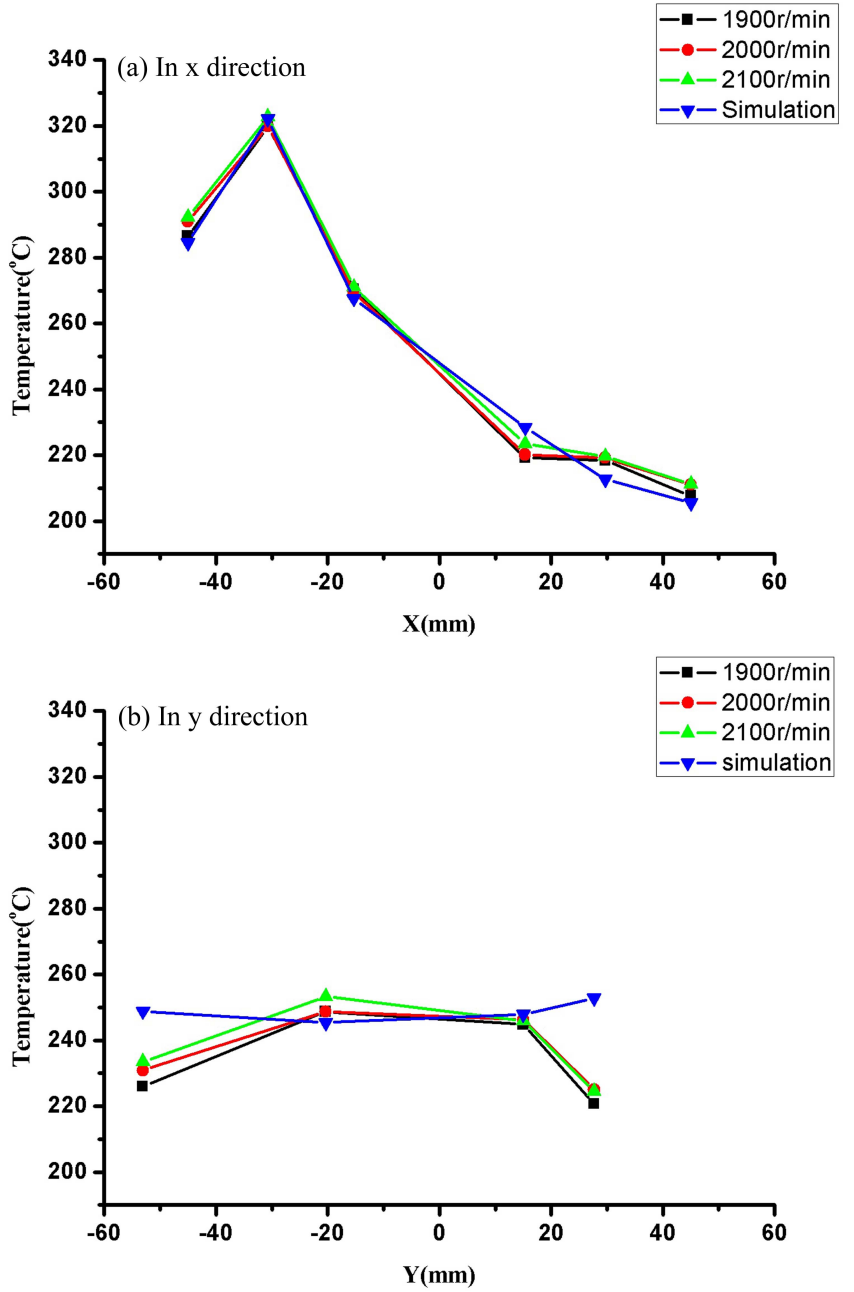

FIG. 6 The comparison between the simulated temperature results with the target ones. (a) The comparison results in $x$ direction; (b) The comparison results in $y$ direction.

\section{THE INTENSITY DISTRIBUTION OF THE SHAPED LASER BEAM AND LASER SHAPING DESIGN}

The real temperature field on the top surface of cylinder head in service conditions is non-uniform field which has particular distribution, and the size range of the temperature field is more than $100 \mathrm{~mm}$. In order to get similar temperature field when using laser beam as heat source, the laser beam shaping technology must to be considered due to the physical feature of original laser beam. The shaped laser beam with certain intensity distribution and its design method are shown in what follows.

\subsection{The intensity distribution of the shaped laser beam}

The intensity distribution of the shaped beam is obtained through a reverse design method with the aid of finite element model (FEM) [8]. Based on the target temperature field, the shaped laser beam with certain intensity distribution is chosen as heat source to irradiate on the top surface of cylinder heat which is shown in Figure 5.The intensity ratio of the three regions is $I$ (Area1) $: I$ (Area2) $: I$ (Area3) $=35: 5: 60$, and $\mathrm{R} 3=10 \mathrm{~mm}, \mathrm{rr} 0=30 \mathrm{~mm}, \mathrm{R} 2=40 \mathrm{~mm}, \mathrm{R} 1=66 \mathrm{~mm}$. The temperature result on key region of particular concern calculated by finite elements model is shown is Figure 6 and Fig- 


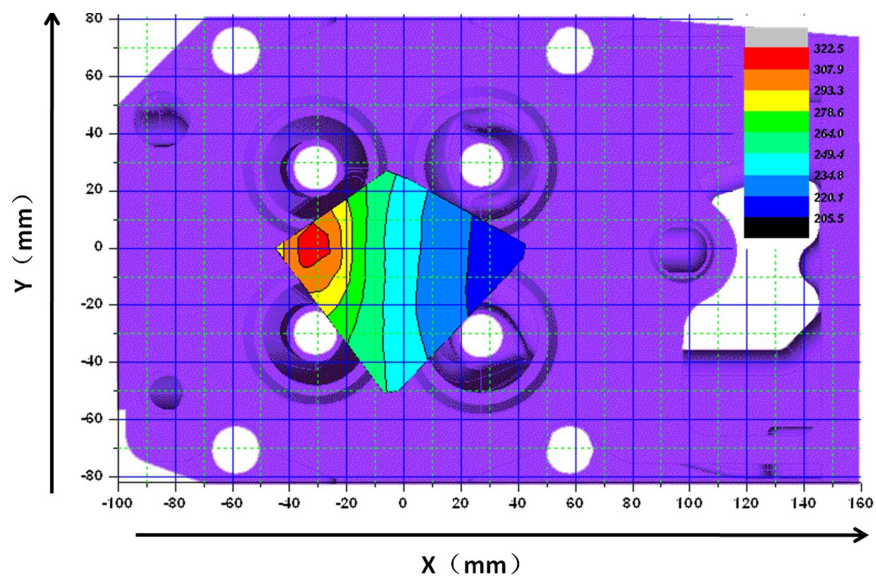

FIG. 7 The simulated temperature distribution by FEM on the combustion surface.

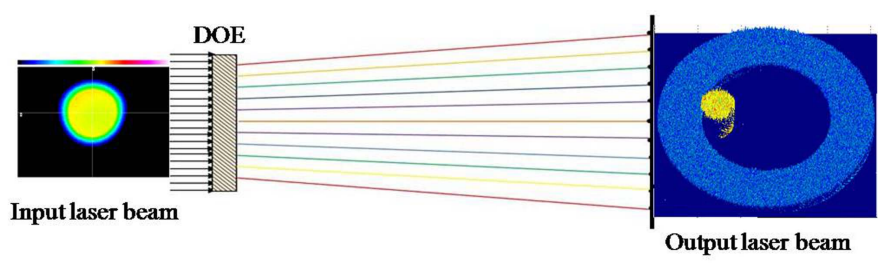

FIG. 8 The sketch of laser shaping design.

ure 7 . We can see that the temperature results in $x$ direction well agree with the target results, but the results close to the region outside in $y$ direction deviate from the target values. However, we focus on the inner area on the bridge zones in the combustion surface, because the inner area on the bridge zones is easily damaged in real service. Therefore, the temperature deviation close to the region outside in y direction has a little effect to the whole temperature distribution on the bridge zones in the key regions. Above all, the temperature results loaded by the shaped laser beam calculated by FEM in the combustion surface on the top surface of the cylinder head are in conjunction with the target temperature field.

\subsection{Laser shaping design}

The shaped laser beam with certain intensity distribution is designed with the aid of diffractive optics element (DOE). The input laser beam irradiates on the DOE, and it is modulated by the phase of the DOE. As a result, the shaped laser beam with desired intensity distribution can be generated at the output plane, and the sketch of laser shaping design is shown in Figure 8 . Because high power laser beams generally have high order transverse modes, the incident laser beam after collimating can be approximately expressed as flattop beams in our study. The diameter of the input plane is $50 \mathrm{~mm}$ and the laser wavelength is $1.064 \mu \mathrm{m}$. The relationship between the input and output plane obeys the equation

$$
\phi_{\text {out }}=N \cdot \frac{\lambda z}{\phi_{\text {in }}}
$$

where $\phi_{\text {out }}$ is the size of the output plane, $\phi_{\text {in }}$ is the size of the input plane, $N$ is the number of sample points, $\lambda$ is the wavelength and $z$ is the distance between the input and output plane. According to the Eq. (5), the sample points of the regions at the output plane can be calculated. The sample points at the input plane is $10000 \times 10000$.

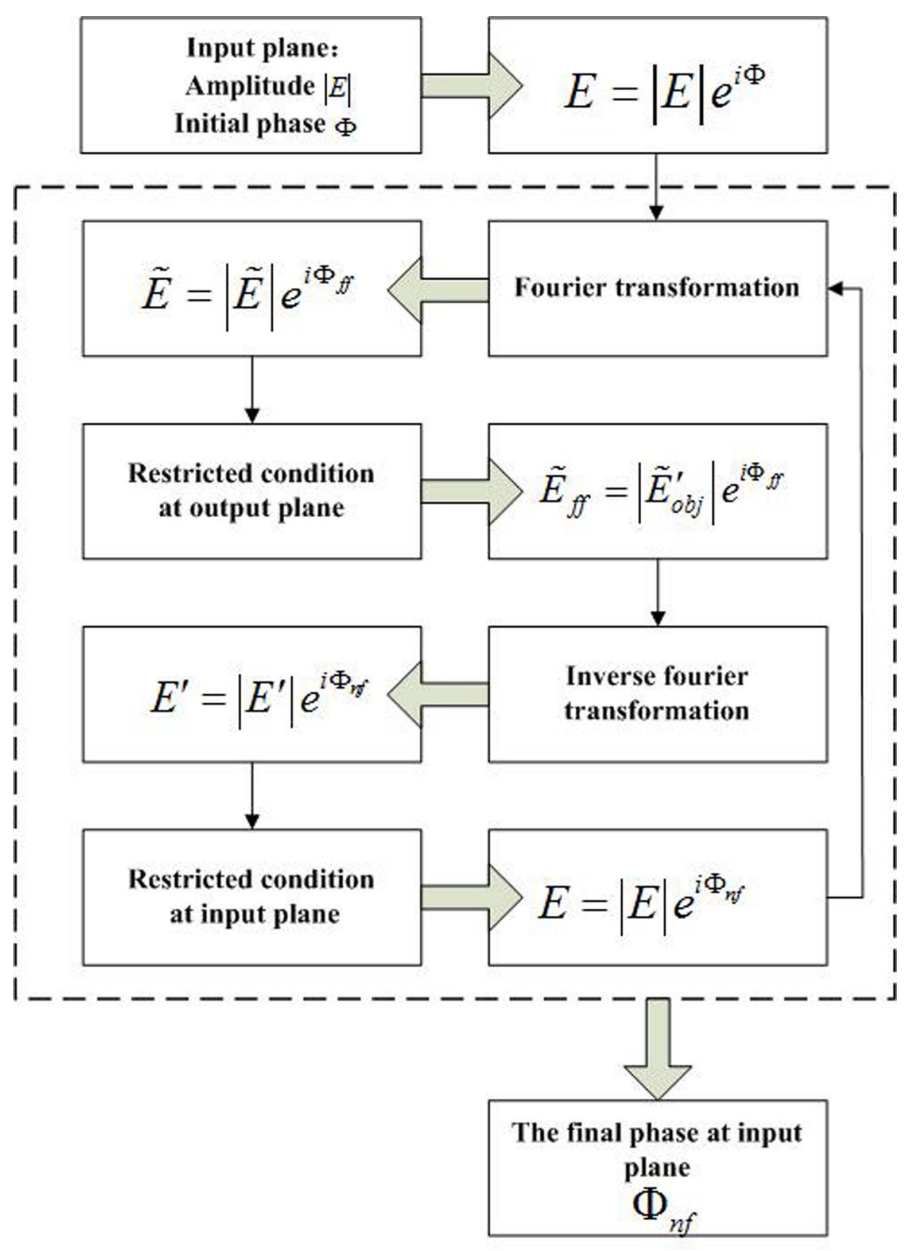

FIG. 9 The phase design process of the DOE.

The phase of the DOE is designed by GS algorithm [20], and the main design process is shown in Figure 9. The output shaped laser beam calculated by GS algorithm is shown in Figure 10. We can see that the calculated result of the shaped laser beam can satisfy the design requirement, and the intensity error is less than $5 \%$. Obviously there also exists large intensity fluctuation in each region, but these fluctuations are acceptable for thermal fatigue test because the average effect of the light power is mainly concerned and the high frequency light can be negligible.

According to the phase date, the DOE is fabricated by very large scale integration technique. The phase of the DOE can be binary, multilevel or continuous. For high efficiency and low costs, multilevel phase modulation of eight-level is chosen. The phase date is quantified to three date groups of $\pi$, $\pi / 2$ and $\pi / 4$. Depending on the three date groups, e-beam direct writing technology is used to create three masks. Subtractive etching process is used, which includes lithography and etching. Lithography process is consisted of treatment of surface cleaning, whirl coating, prebake, exposure and developing. After lithography process, the mask patterns are transformed into the photo-resist layer upon the glass substrate. Reactive ion beam etching is applied to transfer the photoresist pattern onto the substrate. The material of the DOE is quartz, and the etching depth corresponding to the phase $\pi$, $\pi / 2$ and $\pi / 4$ are $1.18 \mu \mathrm{m}, 0.59 \mu \mathrm{m}$ and $0.30 \mu \mathrm{m}$, respectively. After the etching process, the etching depth of the diffractive structure can be measured with a surface profile testing in- 

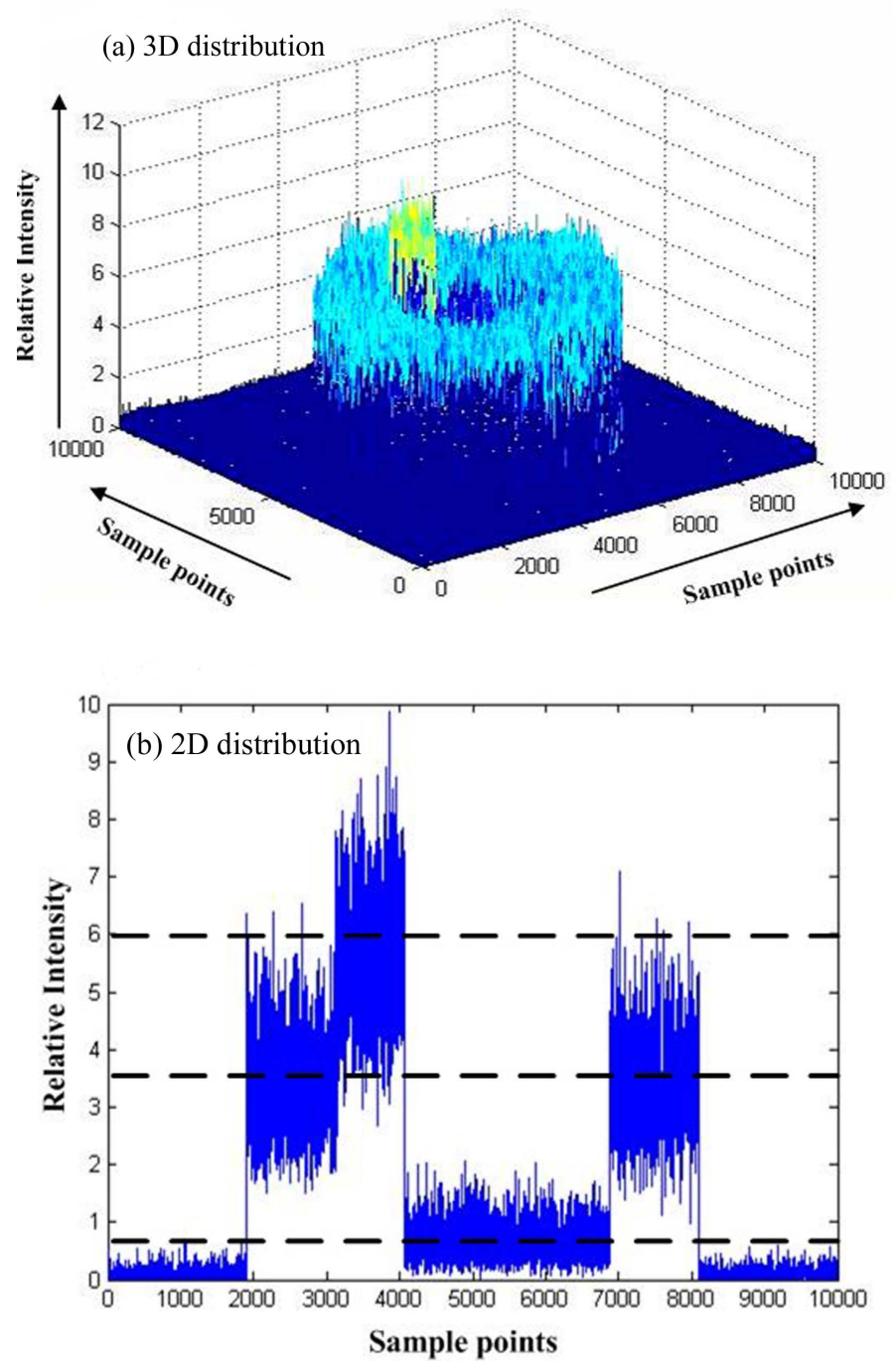

FIG. 10 The calculated intensity distribution of the output shaped laser beam. (a) The 3D calculated intensity distribution; (b) The 2D calculated intensity distribution.

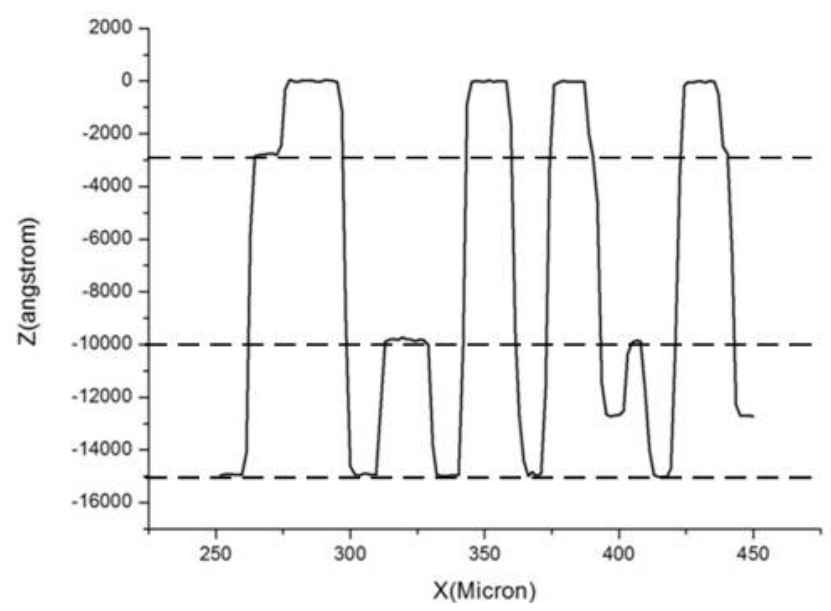

FIG. 11 The etching depth of the DOE.

strument by repeatedly scanning. One of the testing results is shown in Figure 11. The average etching depth corresponding to the phase $\pi, \pi / 2$ and $\pi / 4$ are $1.06 \mu \mathrm{m}, 0.52 \mu \mathrm{m}$ and $0.28 \mu \mathrm{m}$, respectively. The deviations from the desired values corresponding to phase differences are less than $10 \%$.
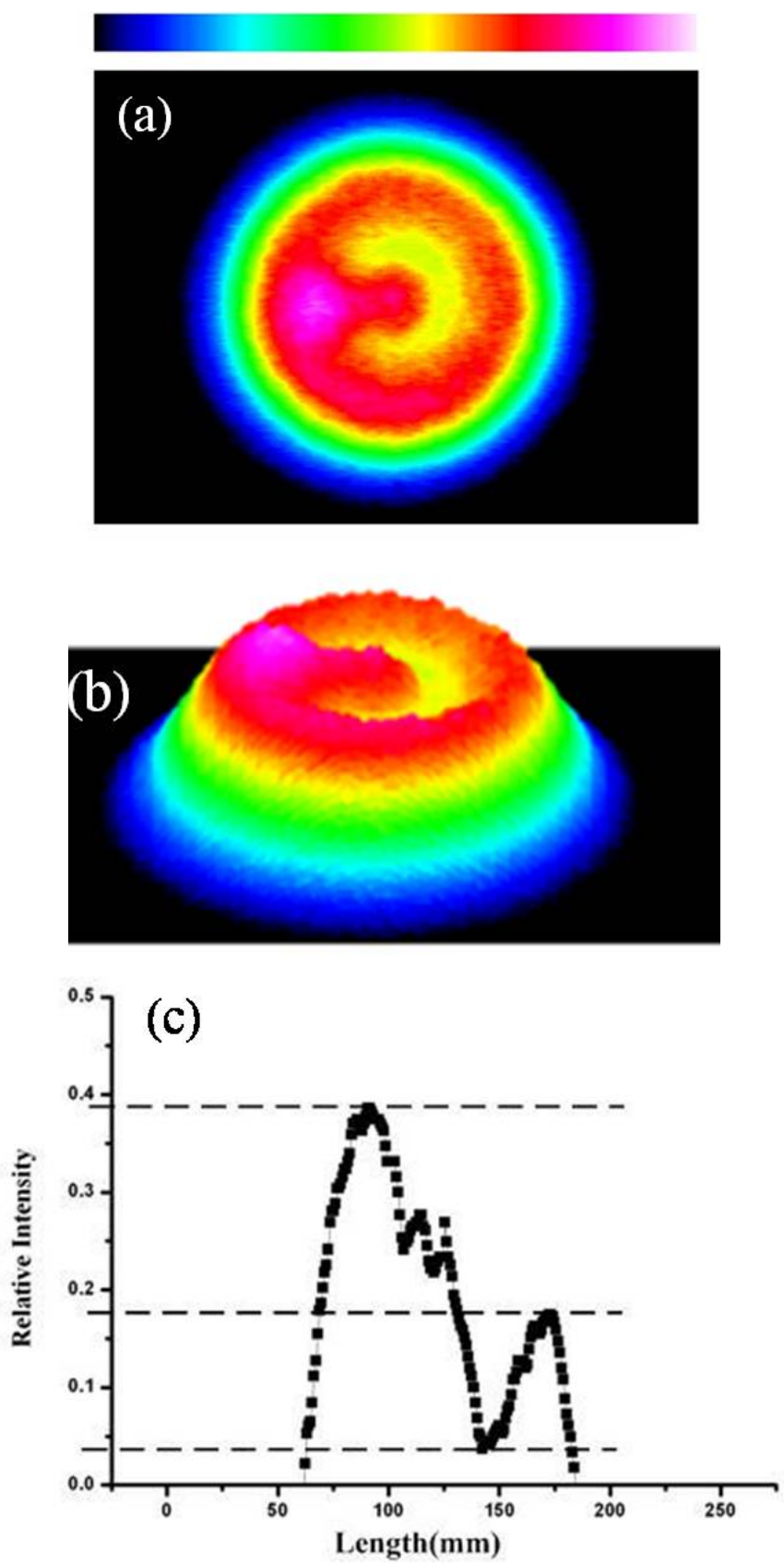

FIG. 12 The intensity distribution of the shaped laser beam detected by laser beam analyser. (a) The 2D intensity distribution; (b) The $3 \mathrm{D}$ intensity distribution; (c) The intensity profile.

\section{EXPERIMENT OF THE TEMPERATURE FIELD LOADED BY THE SHAPED LASER BEAM}

The intensity distribution of the shaped laser beam by the fabricated DOE is detected by laser beam analyser, and the results show in Figure 12. We can see that the regions can meet the requirements and the intensity radio of the three regions is $I($ Area 1$): I($ Area2 $): I($ Area3 $)=30: 5: 65$. The average intensity error is less than $5 \%$, but the zero order intensity is high. For laser thermal fatigue test of cylinder head, high zero order intensity has little effect to the test because the center on the top of the cylinder head is a hole where little energy is absorbed. Above all, the fabricated DOE can transfer the input laser beam into the shaped laser beam with certain intensity distribution. 


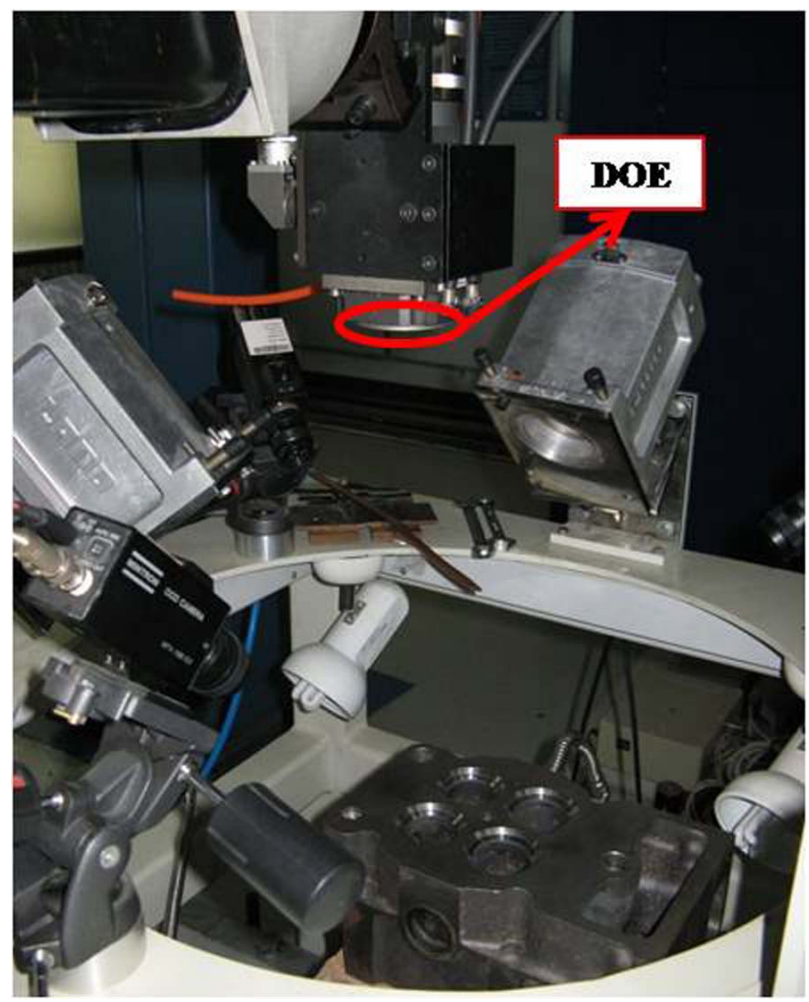

FIG. 13 The experiment equipment.

The fabricated DOE is placed in the laser head which is shown in Figure 13, and the input laser beam irradiates on the DOE. In Figure 13, the infrared pyrometers are mounted on the top of the workbench to sample the surface temperatures at focused spots. The infrared pyrometers work at $2.4 \mu \mathrm{m}$, a wavelength that is not interfered by the working laser. High resolution CCD cameras are employed to sample images of the workbench. Air valve and water valve would be released when auxiliary cooling methods are necessary.

The desired shaped laser beam can be achieved on the top of cylinder head. The distance between the DOE and the top surface of cylinder head is $1 \mathrm{~m}$. The temperature field loaded by the shaped laser beam is measured by infrared pyrometers, and the results are shown in Figure 14. The temperature results show that the temperature field detected by infrared pyrometers is well accordance to the target temperature field distribution, and the temperature error is less than $5 \%$.

When the temperature field induced by the shaped laser beam agrees with the real thermal fatigue ones, thermal high cycle fatigue test and thermal shock test can be carried out by the temperature controlled mode. The temperature controlled mode is achieved by setting up maximum temperature $T_{\max }$ and minimum temperature $T_{\min }$ in one thermal cycle for a given inspection spot. The laser operates in a high power mode till $T_{\max }$ is reached, then shifts to low power mode or no power mode till $T_{\min }$ is reached, forming one thermal cycle. Temperature fluctuation range $\Delta T=T_{\max }-T_{\min }$. In our study, the thermal high cycle fatigue experiments have been performed. The temperature fluctuation is $10-20^{\circ} \mathrm{C}, T_{\max }$ is $330^{\circ} \mathrm{C}$ and $T_{\min }$ is $310^{\circ} \mathrm{C}$. A test result is shown in Figure 15 . Figure 15 is the thermal high cycle temperature test result of the highest temperature spot on the top surface of the cylinder head. The high power of the loaded laser is $3 \mathrm{~kW}$ and the
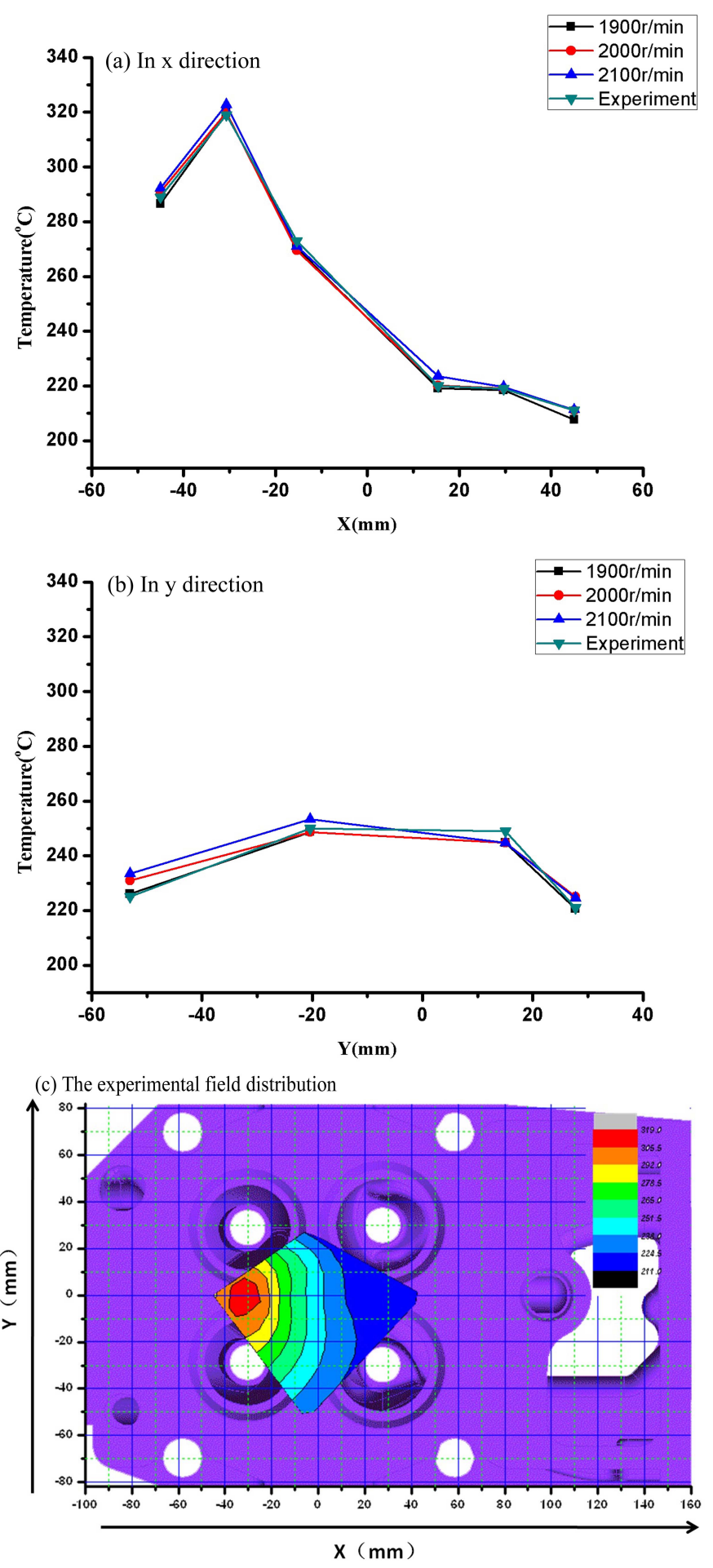

FIC. 14 The temperature field loaded by the shaped laser beam on the combustion surface. (a) The experimental results in $x$ direction; (b) The experimental results in $y$ direction; (c) The experimental field distribution on the combustion surface.

temperature fluctuation is performed with the air cooling. The next thermal fatigue test can be achieved by loading enough thermal cycle which is related to the real service conditions.

Overall, the temperature field produced by the shaped laser beam with certain intensity distribution can match the target temperature distribution on the top surface of cylinder head. This is the first step in the laser thermal fatigue test and the next thermal fatigue test can be carried out based on the temperature distribution. This study can provide the initial foundation for the stress analysis and fatigue life prediction. 


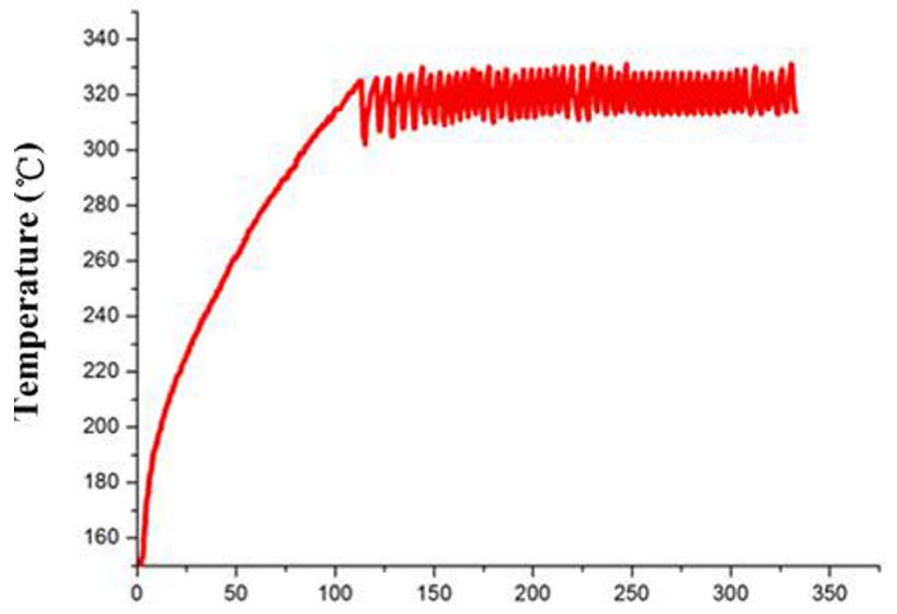

FIG. 15 The preliminary study of thermal high cycle fatigue test.

\section{CONCLUSIONS}

This paper provides an effective method to simulate the real temperature field on the top surface of cylinder head in working service which is measured by thermocouples. The shaped laser beam with certain intensity distribution is chosen as the heat source to irradiate on the top surface of the cylinder head, and the simulated temperature field is calculated by FEM which agrees with the target temperature field. The intensity distribution of the shaped laser beam contains three regions and the intensity ratio is $I$ (Area1) $: I$ (Area2) $: I($ Area3 $)=35$ : $5: 60$. The DOE is designed to transfer the input laser beam into the shaped laser beam based on GS algorithm. The simulated and experimental intensity distribution of the shaped laser beam satisfies the requirements, and the average intensity error is less than $5 \%$. The fabricated DOE is placed in the laser head, and the shaped laser beam irradiates on the top of the cylinder head. In the real temperature test experiments, the temperature field produced by the shaped laser beam can match the target one, and it is a feasible way to simulate the thermal loading in thermal fatigue test which can provides some references for further research work like the thermal fatigue test, stress analysis and life prediction, etc.

\section{References}

[1] M. B. Grieb, H. J. Christ, and B. Plege, "Thermomechanical fatigue of cast aluminium alloys for cylinder head applications - experimental characterization and life prediction," Procedia Eng. 2, 1767-1776 (2010).

[2] T. Beck, D. Löhe, J. Luft, and I. Henne, "Damage mechanisms of cast Al-Si-Mg alloys under superimposed thermalmechanical fatigue and high-cycle fatigue loading," Mater. Sci. Eng A Struct. 468-470, 184-192 (2007).

[3] T. Takahashi, and K. Sasaki, "Low cycle thermal fatigue of aluminum alloy cylinder head in consideration of changing metrology microstructure," Procedia Eng. 2, 767-776 (2010).

[4] W. M. Zhao, and W. Z. Zhang, "Study on thermal fatigue life prediction of cylinder head," Materials Science and Information Technology 433-440, 3-8 (2012).
[5] M. Wu, and J. Campbell, "Thermal fatigue in diesel engine cylinder head castings," Tran. Amer. F. 106, 485-496(1998).

[6] Y. C. Zhou, S. G. Long, and Y. W. Liu, "Thermal failure mechanism and failure threshold of $\mathrm{SiC}$ particle reinforced metal matrix composites induced by laser beam," Mech. Mater. 35, 1003-1020 (2003).

[7] H.-W. Song, G. Yu, J.-S. Tan, L. Zhou, and X.-L. Yu, "Thermal fatigue on pistons induced by shaped high power laser. Part I: Experimental study of transient temperature field and temperature oscillation," Int. J. Heat Mass Tran. 51, 757-767 (2008).

[8] H.-W. Song, G. Yu, A. F. H. Kaplan, J.-S. Tan, and X.-L. Yu, “Thermal fatigue on pistons induced by shaped high power laser. Part II: Design of spatial intensity distribution via numerical simulation," Int. J. Heat Mass Tran. 51, 768-778 (2008).

[9] H.-W. Song, S.-X. Li, L. Zhang, G. Yu, L. Zhou, and J.-S. Tan, “Numerical simulation of thermal loading produced by shaped high power laser onto engine parts," Appl. Therm. Eng. 30, 553-560 (2010).

[10] K. Fuse, T. Hirai, T. Ushiro, T. Okada, K. Kurisu, and K. Ebata, "Design and performance of multilevel phase fan-out diffractive optical elements for laser materials processing," J. Laser Appl. 15, 246-254 (2003).

[11] B. Q. Xu, Z. H. Shen, J. Lu, X. W. Ni, and S. Y. Zhang, "Numerical simulation of laser-induced transient temperature field in film substrate system by finite element method," Int. J. Heat Mass Tran. 46, 4963-4968 (2003).

[12] H. G. Woo, and H. S. Cho, "Three-dimensional temperature distribution in laser surface hardening processes," Proceedings of the Institution of Mechanical Engineers, Part B: Journal of Engineering Manufacture 213, 695-712 (1999).

[13] W. M. Steen, and J. Mazumder, Mathematical modelling of laser/material interactions (European office of Aerospace Research and Development, Final report 1-19, 1983).

[14] T. J. Suleski, and D. C. O'Shea, "Gray-scale masks for diffractiveoptics fabrication: 1. Commercial slide imagers," Appl. Optics 34, 7507-7517 (1995).

[15] M. Schaus, and M. Pohl, "Nd-YAG-Laser simulated thermal shock and thermal fatigue behaviour of railroad steel," Metallurgy 52, 464-470 (1998).

[16] D. M. Zhu, D. S. Fox, R. A. Miller, L. J. Ghosn, and S. Kalluri, "Effect of surface impulsive thermal loads on fatigue behavior of constant volume propulsion engine combustor materials," Surf. Coat. Technol. 188-189, 13-19 (2004).

[17] L.-P. Guo, F.-S. Liu, C.-Y. Wang, and W. Du, "Temperature measurement and numerical study on improving cooling of cylinder head for high power-density," Neiranji Xuebao/Transactions of CSICE (Chinese Society for Internal Combustion Engines) 30, 462-468 (2012).

[18] V. Gupta, and J. Srinivasan, Heat and mass transfer (Tata McGrawHill Publishing Co. Ltd., New Delhi, 1978).

[19] S.-Z. Nie, G. Yu, X.-L. He, C.-Y. Zheng, W.-J. Ning, and S.-X. Li, “Verification of model parameters used in laser thermal fatigue test on cylinder," Guangxue Xuebao/Acta Optica Sinica 31, s100518 (2011) in Chinese.

[20] S.-Z. Nie, J. Yu, Z.-W. Fan, W.-Q. Ge, Y. Liu, and X. Zhang, “Research on laser beam shaping technology using diffractive optics," Guangxue Xuebao/Acta Optica Sinica 33, s105001 (2013) in Chinese. 